\section{An Artefact Suppressing Fast-Recovery Myoelectric Amplifier}

\author{
R. Thorsen
}

\begin{abstract}
An amplifier for recording myoelectric signals using surface electrodes has been developed. The special features are suppression of stimulation artefacts and motion artefacts from electrodes. It is designed for recording of myoelectric signals from a muscle that is being stimulated with short impulses. The artifact suppression is achieved by using fastrecovery instrumentation amplifiers and having a nonlinear feedback loop for automatic compensation of changes in DC-offset.
\end{abstract}

Index Terms-Artifact suppression, electrode offset compensation, electromyography (EMG) amplifier, myoelectric amplifier

\section{INTRODUCTION}

This amplifier is part of a system intended to restore the hand function of spinal cord lesion people [1]. This requires the recording of myoelectric signals from an electrically stimulated muscle. Surface electrodes are used for stimulation and recording. The electrodes are located near each other. Therefore, the myoelectric signal is influenced by stimulation pulses [2] and DC-offset (half-cell potentials) at the recording electrodes. The offset is depending on mechanical actions on the electrode [3] (motion artifacts).

A typical electromyography (EMG) amplifier is configured with a high-pass filter at the input stage [4]-[6] to remove DC-offset. Such a high-pass filter (see Fig. 1) causes transients from the stimulation impulses to saturate the subsequent stages and, thus, extend the duration of the stimulation artifacts and block the myoelectric signal. To deal with this problem a switch [7], [8] or a sample-hold circuit [9] can be applied before the filter, or the filter cutoff frequency can be raised $(1 \mathrm{kHz})$, well above the stimulation frequency [10]. Switches (CMOS) can cause a charge injection in the signal path, which causes new artifacts. Furthermore a high cutoff frequency is inadequate for recording surface myoelectric signals for which it is commonly accepted that the information is present in the frequencies below $500 \mathrm{~Hz}$ [11], [12].

A linear feedback loop [13], [14] can compensate the DC offset but will behave like the amplifier in Fig. 1 with respect to stimulation artifacts. A high-pass filter with very low cutoff frequency minimizes the artifact problem but will give rise to slow adaptations to changes in the DC-offset. This can be solved by adding a manually operated [15] or threshold-controlled [16] switch for temporary change of the filter time constant. The concept described in the following is an approach to minimize the stimulation artifacts while maintaining the low cutoff frequency together and a fast recovery. It uses DC compensation by two feedback loops combining artifact suppression and fast DC offset compensation. The recovery time is here defined, as the time from the end of stimulation pulse to the circuit has a linear response. The amplifier possesses the ability of accommodate sudden changes in the differential DC offset at the input.

Manuscript received November 20, 1997; revised January 5, 1999. This work was supported by the Academy of Technical Sciences, Denmark.

The author is with the University of Twente, Department of Signal and Systems-BME, P.O. Box 217, 7500 AE Enschede, The Netherlands (e-mail: r.a.thorsen@el.utwente.ml).

Publisher Item Identifier S 0018-9294(99)03357-1.

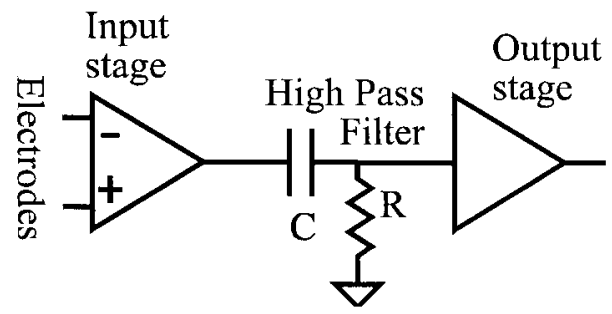

Fig. 1. Simplified schematic of a typical conventional myoelectric amplifier

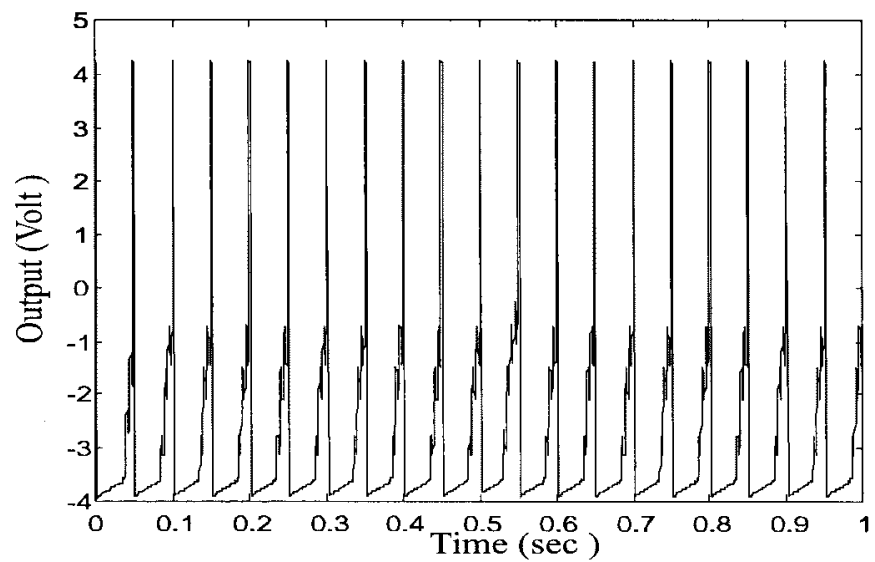

Fig. 2. Response from a conventional amplifier with the input of Fig. 4.

\section{METHOD}

The simplified schematic for the developed amplifier suited for myoelectric controlled functional electric stimulation is shown at Fig. 3. It is designed as a $\pm 3 \mathrm{~V}$ system with a total gain of $74 \mathrm{~dB}$ and a small signal high-pass filter cutoff frequency at $8 \mathrm{~Hz}$. The system is designed for typical magnitudes of the signal components as follows.

- Myoelectric signals: Less than $0.5 \mathrm{mV}$, frequency range: $20-500$ $\mathrm{Hz}$.

- Stimulation artefacts: Less than $\pm 3 \mathrm{~V}, 1 \mathrm{~ms}$ duration, $20 \mathrm{~Hz}$ repetition rate.

- Electrode offset changing within the range of $\pm 0.1 \mathrm{~V}$ and amplifier recovery time less than $0.1 \mathrm{~s}$.

The theory of operation is as follows.

The signal from the electrodes is clamped between the power supplies, through a resistor diode network (not shown), to protect the instrumentation amplifier IC1. The resulting offset at the output of IC 1 is compensated by the output, $V_{2}$, of IC 3 through the resistors $R_{3}$ and $R_{2}$. These resistors are selected to be equal for the best compromise between minimal signal attenuation and maximal offset compensation range. An output range of $\pm 3 \mathrm{~V}$ of IC1 allows $20 \mathrm{~dB}$ gain in IC1. It is desirable to have highest possible amplification in IC1 since this determines the overall common mode rejection ratio (CMRR) and CMRR for this type of instrumentation amplifiers increase with increasing gain. Resistors $R_{3}$ and $R_{2}$ gives $6 \mathrm{~dB}$ damping and IC2 is, thus, chosen to have $60 \mathrm{~dB}$ gain to provide at total gain of $74 \mathrm{~dB}$. The stimulation artifacts are saturating both IC1 and IC2. For this reason they are chosen be fast-recovery circuits $(<10 \mu \mathrm{s})$. The network around IC3 is a two-path DC feedback, with pulse suppression. 


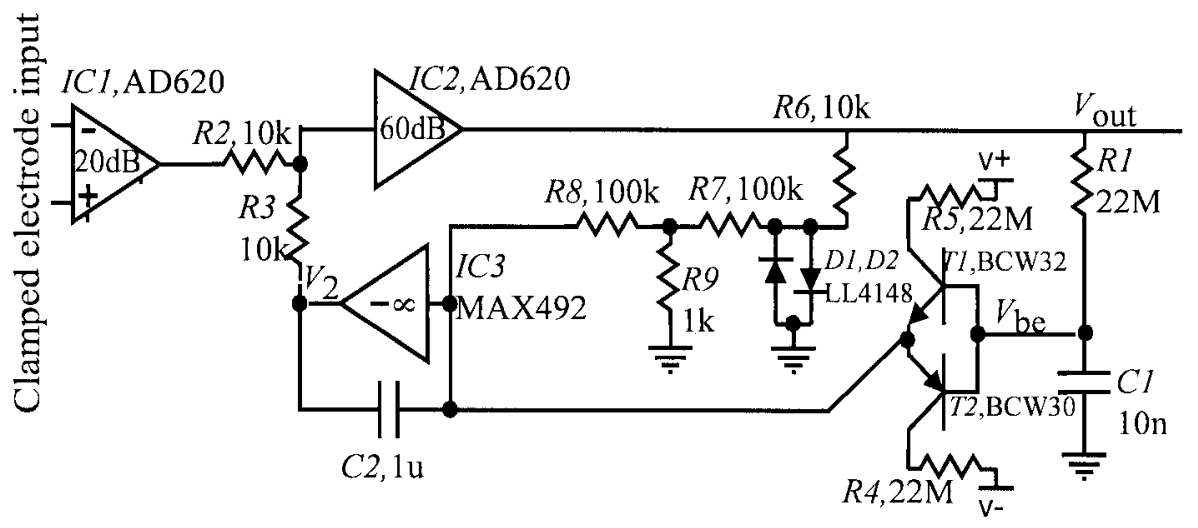

Fig. 3. Simplified schematic of the amplifier (patent pending).

An offset change of more than $0.1 \mathrm{mV}$ ( $V_{b e, \text { on }}$ divided by the gain) will be compensated by the $R_{1}, C_{1}, T_{1,2}, C_{2}$ path. Time constant $R_{1} C_{1}$ is chosen such that the voltage over $C_{1}$ will not exceed the basis-emitter on voltage $V_{b e}$, on for the transistors during pulses. To find the time constant $R_{1} C_{1}$ of the low-pass filter it is assumed that the transistors are switches that are open below the $V_{b e}$, on voltage of about $\pm 0.6 \mathrm{~V}$. The operational amplifier IC3 will keep the emitter voltage at zero. If a DC-offset saturates the amplifier, the output of IC2 ( $V_{\text {out }}$ ) will be clamped to $\pm 3 \mathrm{~V}$, and the basis-emitter voltage at $V_{b e}$ will change accordingly to

$$
V_{\text {be }}=V_{\text {out }}\left(1-\exp \left[\frac{-t}{R_{1} C_{1}}\right]\right) .
$$

This can be reformulated to determine $R_{1} C_{1}$ for a given time $t$, after which DC compensation should start

$$
R_{1} C_{1}=\frac{-t}{\ln \left(1-V_{b e, \text { on }} / V_{\text {out }}\right)} .
$$

A selected value of $t=50 \mathrm{~ms}$, thus, gives $R_{1} C_{1}=0.22 \mathrm{~s}$. The transistors operate as a switch which begins to charge $C_{2}$ when the potential over $C_{1}$ exceeds an absolute value of the knee voltage of the transistors $V_{b e, \text { on }}(0.6 \mathrm{~V})$. Assuming that the basis current in the transistors can be neglected and that they act as a switch, the current into the capacitor $C_{2}$ is determined by resistor $R_{5}$, respectively, the identical $R_{4}$. The time constant $R_{5} C_{2}$ is given by

$$
R_{5} C_{2}=\frac{V_{R 5}}{\Delta V_{2} / t_{r}}
$$

where $V_{R 5}$ is the voltage over $R_{5}$ which is $3 \mathrm{~V}$ (transistor collectoremitter resistance can be neglected) and $\Delta V_{2} / t_{r}$ is the compensation rate. Saturation of IC2 (with gain 500) needs a minimum change of $\Delta V_{2}=3 \mathrm{~V} / 500=6 \mathrm{mV}$. Choosing $t_{r}=50 \mathrm{~ms}$ gives $R_{5} C_{2}=25$ ms. With $C_{2}=1 \mu \mathrm{F}$ the nearest standard resistor value gives $R_{5}=22 \mathrm{M} \Omega$. It is important that IC2 is an amplifier that does not reverse the output when over-steered.

For low-level signals $(<0.1 \mathrm{mV})$, the amplifier has high-pass filter characteristic. Network $R_{6}, R_{7}, R_{8}, R_{9}, C_{2}$, and IC3 determines this. Diodes $D_{1}$ and $D_{2}$ clamps the feedback signal, limits the (dis)charging of $C_{2}$ caused by pulses and thus minimize transients caused by stimulation pulses. The transfer function in the linear range can be found to be

$$
\begin{aligned}
H(s) & =\frac{V_{\text {out }}}{V_{\text {in }}}=G_{1} \frac{s\left(k C_{2} R_{8}\right)}{1+s\left(2 k C_{2} R_{8}\right) / G_{2}} \\
k & =\left(R_{6}+R_{7}\right)\left(1 / R_{8}+1 / R_{9}\right)+1 .
\end{aligned}
$$

Here, $G_{1}$ and $G_{2}$ are the gains of IC1, respectively, IC2. For the present amplifier the component values have been chosen so that the

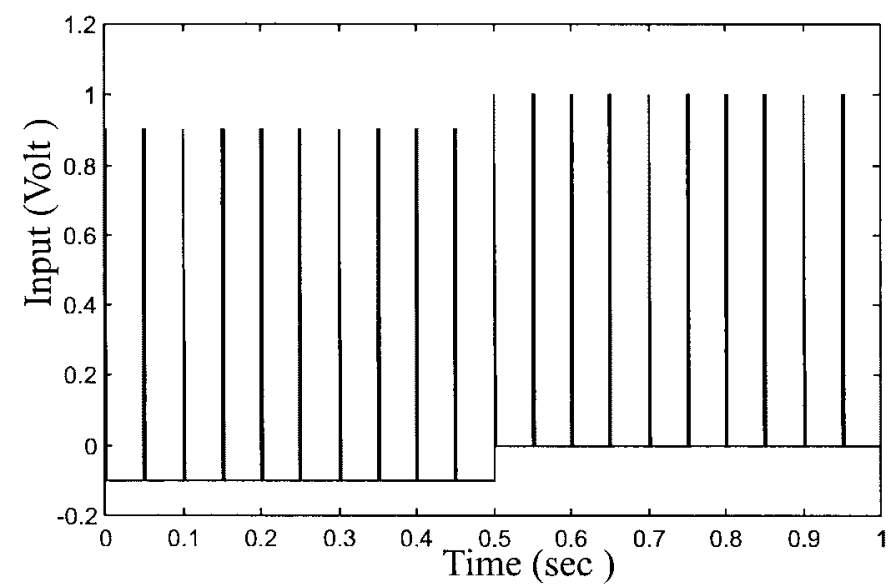

Fig. 4. Excitation test signal.

myoelectric signal frequency is high-pass filtered at $8 \mathrm{~Hz}$ and that large DC-offset compensation starts after $50 \mathrm{~ms}$ which is equivalent to one stimulation period at $20 \mathrm{~Hz}$. This ensures that the stimulation pulses and compound muscle action potentials are not initiating the DC-offset compensation.

\section{RESULTS}

The circuit has been build and the functionality has been proved to comply with calculations and SPICE simulations. For convenience the SPICE simulations are shown in the following. (The simulation is based on a $\pm 5 \mathrm{~V}$ system using LM324 op-amp model.)

The test signal is a sum of:

- $0.1 \mathrm{mV}, 200 \mathrm{~Hz}$ sine wave representing the myoelectric signal;

- $1 \mathrm{~V}, 2 \mathrm{~ms}$ pulse repeated 20 times/s representing the stimulation artefacts;

- $-0.1 \mathrm{~V}, 500 \mathrm{~ms}$ pulse representing a change in electrode offset.

The test signal can be seen on Fig. 4. A $74 \mathrm{~dB}$ amplifier, as in Fig. 1, with a $15 \mathrm{~Hz}$ first-order high-pass filter at the input would be saturated by this signal, as shown in Fig. 2. Thus, the myoelectric signal will not be amplified. The amplifier in Fig. 3 suppresses both the stimulation pulses and the offset change, permitting amplification of the myoelectric signal. The output of a SPICE simulated circuit with the test input can be seen on Fig. 5. The adjustment for the change in offset starts after $50 \mathrm{~ms}$ and is completed within another $100 \mathrm{~ms}$. This allows myoelectric signals to pass through the amplifier $150 \mathrm{~ms}$ after an offset change. This is, of course, dependent on the 


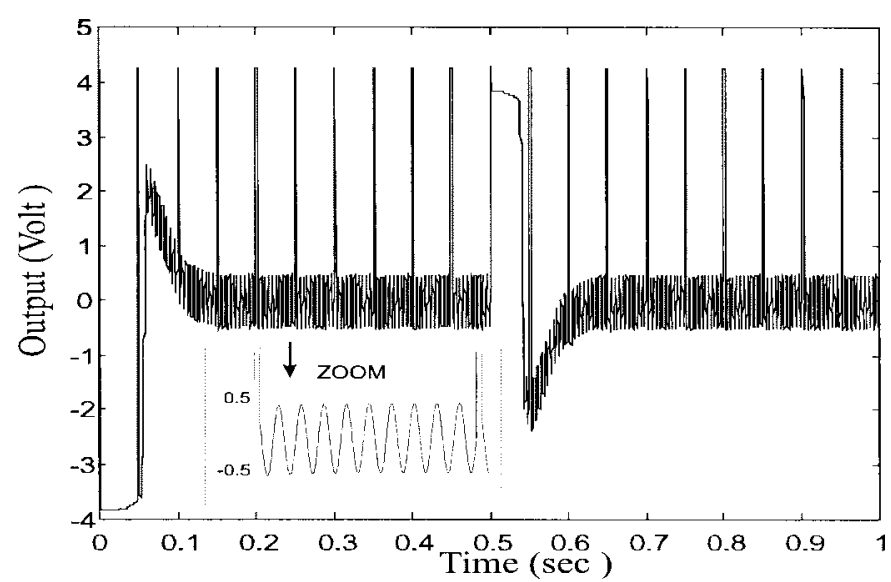

Fig. 5. Amplifier output (SPICE simulation).

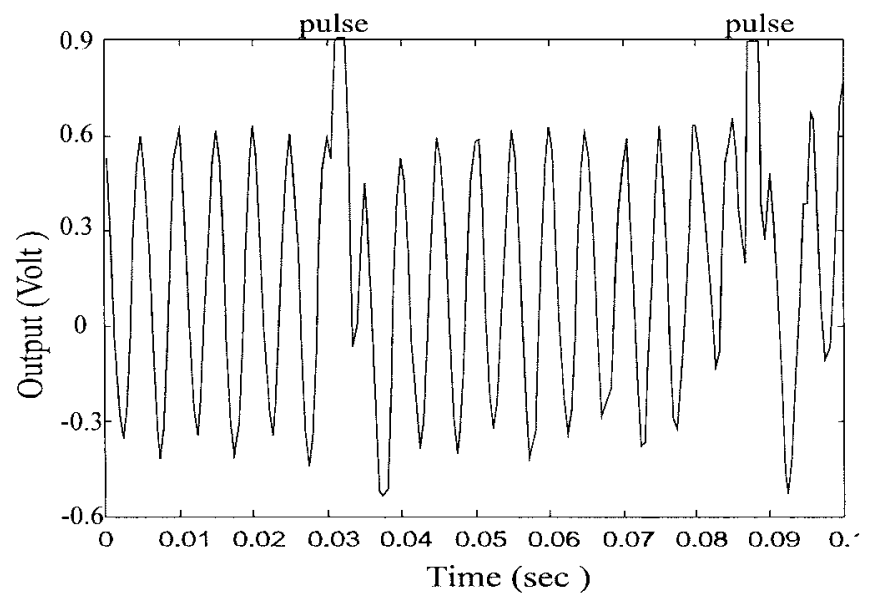

Fig. 6. Measured output from the amplifier. The input is a $200 \mathrm{~Hz}, 0.1 \mathrm{mV}$ sine superimposed with a $1 \mathrm{~V}$ stimulation pulse. The sampling frequency is $2 \mathrm{kHz}$.

magnitude of the change. The zoom shows that the stimulation does not affect the signal in between the stimulation pulses.

To verify the theoretical and simulated results the amplifier has been realized in hardware. A setup where one input is connected to two signal generators and the other input grounded has been used for the testing. The signal generators are connected to generate the sum of a $0.1 \mathrm{mV}$ sine and the $1 \mathrm{~V}$ pulse. A recording of the output is shown in Fig. 6. The signal is sampled at $2 \mathrm{kHz}, 10 \mathrm{~b}$. The slight distortion of the signal shown is a result of $50 \mathrm{~Hz}$ powerline interference and the sampling resolution. The noise level with both inputs grounded is measured to be $3 \mu V_{R T I, P P}$. The impedance of the test signal is much lower than the amplifier input impedance and the noise is mainly due to the quality of available signal generators. The overall gain has been measured to be $74 \mathrm{~dB} \pm 1 \mathrm{~dB}$. The measurements have shown that the amplifier possesses the desired characteristics. Offset compensation has been verified qualitatively by changing offset on the pulse generator. As it can be seen there is an offset of the output signal of ca. $0.1 \mathrm{mV}$, which can be due to offset in IC3 (MAX492).

\section{CONCLUSION}

The amplifier is an AC-signal amplifier, which does not saturate by short stimulation artefacts. Changes in the DC-offset at the input are compensated within a short period determined by the time constant of a RC circuit. It can be useful for applications where electrodes are used to record bioelectrical signals from tissue which is stimulated electrically by short impulses.

\section{ACKNOWLEDGMENT}

This work was carried out at The Department of Mathematical Modeling, The Technical University of Denmark, and the prototypes were produced by Asah Medico A/S, Valseholmen 11-13, DK-2650 Hvidovre, Denmark.

\section{REFERENCES}

[1] R. Thorsen, F. Biering-Sørensen, O. T. Andersen, and S. D. Hansen, "Myoelectric signals from paretic muscles controlling electrical stimulation of the same muscle," in 4th Topical Workshop of the Concerted Action, RAFT. Berlin, Germany: Springer-Verlag, 1996, pp. 373-376.

[2] R. Merletti, M. Knaflitz, and C. J. DeLuca, "Electrically evoked myoelectric signals," Crit. Rev. Biomed. Eng., vol. 19, pp. 293-340, 1992.

[3] J. G. Webster, "Reducing motion artifacts and interference in biopotential recording," IEEE Trans. Biomed. Eng., vol. 31, pp. 823-826, 1984.

[4] H. E. Hall and W. A. Munday, "Electromyographic treatment device," U.S. Patent, 53000961994.

[5] C. J. Ylvisaker, "Patient initiated response method," U.S. Patent, 45820491986

[6] G. N. Saridis and T. P. Goothe, "EMG pattern analysis and classification for a prosthetic arm," IEEE Trans. Biomed. Eng., vol. 6, pp. 403-412, 1982.

[7] J. Mizrahi, M. Levy, H. Ring, E. Isakov, and A. Liberson, "EMG as an indicator of fatigue in isometrically FES-activated paralyzed muscles," IEEE Trans. Rehab. Eng., vol. 2, pp. 57-65, 1994.

[8] J. Minzly, J. Mizrahi, N. Hakim, and A. Liberson, "Stimulus artefact suppressor for EMG recording during FES by a constant-current stimulator," Med. Biol. Eng. Comput., vol. 31, pp. 72-75, 1993.

[9] D. C. Howson and J. E. Heule, "Electronic circuit permitting simultaneous use of stimulating and monitoring equipment," U.S. Patent, 42352421980

[10] M. K. Haugland and J. A. Hoffer, "Artifact-free sensory nerve signals obtained from cuff electrodes during functional electrical stimulation of nearby muscles," IEEE Trans. Rehab. Eng., vol. 2, pp. 37-40, Mar. 1994.

[11] M. Bilodeau, M. Cincera, S. Gervais, A. B. Arsenault, D. Gravel, Y. Lepage, and P. McKinley, "Variations in the shape of the EMG power spectrum during an increasing force level," in Proc. Annu. Conf. on Engineering in Medicine and Biology, vol. 15, pp. 1206, 1993.

[12] E. Kwatny, D. H. Thomas, and H. G. Kwatny, "An application of signal processing techniques to the study of myoelectric signals," IEEE. Trans. Biomed. Eng., vol. BME-17, pp. 303-313, 1970.

[13] H. W. Smit, K. Verton, and C. A. Grimbergen, "A low-cost multichannel preamplifier for physiological signals," IEEE Trans. Biomed. Eng., vol. BME-4, pp. 307-310, 1987.

[14] G. Hamstra, A. Peper, and C. Grimbergen, "Low-power, low-noise instrumentation amplifier for physiological signals.," Med. Biol. Eng. Comput., vol. 22, no. 3, pp. 272-274, May 1984.

[15] R. v. Heuningen, H. G. Goovaerts, and F. R. d. Vries, "A low noise isolated amplifier system for electrophysiological measurements: Basic considerations and design," Med. Biol. Eng. Comput., vol. 22, no. 1, pp. 77-85, Jan. 1984.

[16] D. D. Walker and J. Kimura, "A fast-recovery electrode amplifier for electrophysiology," Electroencephal. Clin. Neurophysiol., vol. 45, pp. 789-792, 1978. 